\title{
Application of a small molecule inhibitor screen approach to identify CXCR4 downstream signaling pathways that promote a mesenchymal and fulvestrant-resistant phenotype in breast cancer cells
}

\author{
MARGARITE D. MATOSSIAN ${ }^{1}$, STEVEN ELLIOTT ${ }^{1}$, LYNDSAY V. RHODES $^{2}$, \\ ELIZABETH C. MARTIN ${ }^{3}$, VAN T. HOANG ${ }^{1}$, HOPE E. BURKS ${ }^{1}$, WILLIAM J. ZUERCHER ${ }^{4}$, \\ DAVID H. DREWRY ${ }^{4}$, BRIDGETTE M. COLLINS-BUROW ${ }^{1}$ and MATTHEW E. BUROW ${ }^{1}$ \\ ${ }^{1}$ Department of Medicine, Section of Hematology and Oncology, Tulane University School of Medicine, \\ New Orleans, LA 70112; ${ }^{2}$ Department of Biology, Florida Gulf Coast University, Fort Myers, FL 33965; \\ ${ }^{3}$ Department of Biological and Agricultural Engineering Biology, Louisiana State University, \\ Baton Rouge, LA 70803; ${ }^{4}$ Structural Genomics Consortium, Eshelman School of Pharmacy, \\ University of North Carolina, Chapel Hill, NC 27599, USA
}

Received March 7, 2020; Accepted November 30, 2020

DOI: $10.3892 / \mathrm{ol} .2021 .12641$

\begin{abstract}
Chemokine receptor 4 (CXCR4) and its ligand stromal-derived factor 1 (SDF-1) have well-characterized functions in cancer metastasis; however, the specific mechanisms through which CXCR4 promotes a metastatic and drug-resistant phenotype remain widely unknown. The aim of the present study was to demonstrate the application of a phenotypic screening approach using a small molecule inhibitor library to identify potential CXCR4-mediated signaling pathways. The present study demonstrated a new application of the Published Kinase Inhibitor Set (PKIS), a library of small molecule inhibitors from diverse chemotype series with varying levels of selectivity, in a phenotypic medium-throughput screen to identify potential mechanisms to pursue. Crystal violet staining and brightfield microscopy were employed to evaluate relative cell survival and changes to cell morphology in the screens. 'Hits' or lead active compounds in the first screen were PKIS inhibitors that reversed mesenchymal morphologies in CXCR4-activated breast cancer cells without the $\mathrm{COOH}$-terminal domain (MCF-7-CXCR4- $\triangle \mathrm{CTD}$ ) and in the phenotypically mesenchymal triple-negative breast cancer cells (MDA-MB-231, BT-549 and MDA-MB-157),
\end{abstract}

Correspondence to: Dr Matthew E. Burow, Department of Medicine, Section of Hematology and Oncology, Tulane University School of Medicine, 1430 Tulane Avenue, New Orleans, LA 70112, USA

E-mail: mburow@tulane.edu

Key words: chemokine receptor 4, breast cancer, medium-throughput screen, published kinase inhibitor set, small molecule inhibitors, fulvestrant resistance, metastasis, mesenchymal phenotype, triple-negative breast cancer used as positive controls. In a following screen, the phenotypic and cell viability screen was used with a positive control that was both morphologically mesenchymal and had acquired fulvestrant resistance. Compounds within the same chemotype series were identified that exhibited biological activity in the screens, the 'active' inhibitors, were compared with inactive compounds. Relative kinase activity was obtained using published datasets to discover candidate kinase targets responsible for CXCR4 activity. MAP4K4 and MINK reversed both the mesenchymal and drug-resistant phenotypes, NEK9 and DYRK2 only reversed the mesenchymal morphology, and kinases, including ROS, LCK, HCK and LTK, altered the fulvestrant-resistant phenotype. Oligoarray experiments revealed pathways affected in CXCR4-activated cells, and these pathways were compared with the present screening approach to validate our screening tool. The oligoarray approach identified the integrin-mediated, ephrin B-related, RhoA, RAC1 and ErbB signaling pathways to be upregulated in MCF-7-CXCR4- $\triangle$ CTD cells, with ephrin B signaling also identified in the PKIS phenotypic screen. The present screening tool may be used to discover potential mechanisms of targeted signaling pathways in solid cancers.

\section{Introduction}

Screening modalities to determine candidate downstream targets and signaling pathways after novel and influential proteins/pathways identified are limited by availability and utility. Small molecule inhibitor libraries in cancer research introduce a unique modality to screen for various biologic endpoints, including drug sensitivity, cell morphology, cell proliferation, and survival (1). The Published Kinase Inhibitor Set (PKIS) is an example of such a library; PKIS1 and PKIS2 are collections of ATP-competitive kinase inhibitors representing dozens of chemotypes (2). The described inhibitors 
have a range of selectivity profiles against various kinase targets (3) and can thus be used in a screening approach to identify candidate kinases targets or downstream signaling pathways to pursue in further mechanistic studies for select genes of interest. Previously, we demonstrated the successful application of the PKIS in a morphology-based screen as a starting point to discover kinase targets and signaling pathways that drove a specific phenotype in TNBC cells (4).

In this report, we utilized genetically modified breast cancer cell lines to demonstrate the application of this phenotypic screening approach to identify candidate targets to pursue for potential drug discovery applications. For these experiments, we focused on studying the downstream effects of over-activation of the CXC chemokine receptor 4 (CXCR4). Chemokine-mediated signaling processes have integral roles in cancer development and metastasis $(5,6)$. While chemokines can bind to various chemokine receptors, CXCR4 is unique because it exclusively binds to the CXCL12 chemokine, also known as stromal cell-derived factor-1 (7). CXCR4 is a $\mathrm{G}$ protein receptor that subsequently activates phospholipase C- $\beta$ and phosphatidylinositol-3-kinase, or PI3K. These signaling events cause downstream activation of protein kinase $\mathrm{C}$ and mitogen-activated protein kinase, which leads to cell migration (8). Small molecule therapies targeting CXCR4 are currently being investigated as anti-cancer therapeutics $(9,10)$, providing evidence for CXCR4 as a viable target in endocrine therapy-resistant breast cancer $(11,12)$. CXCR4 is expressed in many different cancer types $(7,13)$, and its expression is associated with higher-grade cancers (14). CXCR4 has been implicated as a prognostic marker in breast cancer and is associated with worse prognoses $(15,16)$. In triple-negative breast cancer (TNBC), a subtype lacking hormone receptors or HER2/Neu amplification, activated CXCR4 is present in $75 \%$ of TNBC tumors, as was evaluated in microarray analysis $(17,18)$. CXCR4 expression drives breast cancer cell invasion and metastasis $(13,19-21)$. Furthermore, the CXCR4-SDF-1 signaling axis regulates the activity of circulating tumor cells in primary breast cancer (22). In metastasis, cells acquire characteristics that drive an invasive and migratory phenotype in a process known as epithelial-mesenchymal transition (EMT) (23). In EMT, cancer cells that are epithelial-shaped and have epithelial molecular phenotypes acquire mesenchymal molecular features which induce a change in cell morphology to a more fibroblastic and stellate-appearing shape $(23,24)$. Acquisition of a mesenchymal phenotype drives cell invasion and migration through the extracellular matrix and intravasate into surrounding vasculature to disseminate to distal tissue sites $(25,26)$.

CXCR4 activates signaling pathways that drive tumor growth and angiogenesis. CXCR4 is positively upregulated by the hypoxia-inducible factor- $1 \alpha$ and growth factors (FGF, VEGF, EGF) (27). The COOH-terminal domain (CTD) mediates receptor desensitization and downregulation (27), and truncation of this domain $(\triangle \mathrm{CTD})$ results in sensitization and upregulation of the receptor. The CTD domain is necessary to drive a mesenchymal cell morphology and cell motility through CXCR4 signaling (27-29): $\triangle \mathrm{CTD}$, and not CXCR4 overexpressing cells, downregulated epithelial protein expression (CDH1, ZO1), decreased cell-cell contact, and increased cell migration (27).
CXCR4 activation is additionally associated with endocrine therapy resistance through the downregulation of estrogen receptor expression (30). CXCR4 signaling is implicated in other areas of drug resistance: In breast cancer, CXCR4 silencing sensitizes TNBC cells to cisplatin therapy (31), silencing of CXCR4 and SDF-1 sensitizes breast cancer cells to paclitaxel (32), and CXCR4 inhibition abrogates trastuzumab resistance in HER2-positive breast cancer (33). We previously demonstrated that CXCR4 expression mediates estrogen-independent tumorigenesis, metastasis, and resistance to endocrine therapies through increased MAPK signaling $(34,35)$. CXCR4 activates ER-mediated gene transcription through phosphorylation of ER $\beta$ by MAPK family members (36), inducing estrogen independence in MCF-7-CXCR4 cells. Dubrovska et al found that CXCR4 maintains a cancer stem cell-like progenitor population in tamoxifen-resistant MCF-7 cells (37). Together, these findings support a role for CXCR4 activation in endocrine therapy resistance in addition to driving a mesenchymal and migratory phenotype.

Downstream signaling pathways of CXCR4 that are responsible for these phenotypic changes remain widely unknown. To address this knowledge gap, we employed the PKIS library in a medium-throughput phenotypic screen using MCF-7 parental cells (MCF-7), MCF-7 cells with constitutively active CXCR4 expression (MCF-7-CXCR4- $\triangle \mathrm{CTD}$ ), fulvestrant resistant MCF-7 cells (MCF-7-FR), and TNBC cell lines (BT-549, MDA-MB-231). We then compared relative kinase activity of compounds within the same chemotype series that were active or inactive in the screens. Our goal was to identify candidate signaling pathways responsible for the observed mesenchymal and fulvestrant-resistant phenotype of MCF-7-CXCR4- $\triangle \mathrm{CTD}$ cells. This aim of this study was to demonstrate the utility in using a phenotypic screening approach with small molecule kinase inhibitors to identify potential pathways and targets downstream to pursue in CXCR4-activated breast cancer cells. Future experiments will be required to validate and interrogate the kinase pathway leads identified in this screen.

\section{Materials and methods}

Cell culture. Human MDA-MB-157, MDA-MB-231 and BT-549 cells were acquired from the American Type Culture Collection (ATCC). Human MCF-7 cells used for stable transfection of CXCR4 were generously provided to our lab by Louisa Nutter (University of Minnesota, Minneapolis, MN, USA). Cells were maintained in DMEM supplemented with $10 \%$ fetal bovine serum, $1 \%$ non-essential amino acids (NEAA) (Caisson Labs), MEM amino acids (Invitrogen; Thermo Fisher Scientific, Inc.), antibiotic-antimycotic solution (100 U/ml; Caissan Labs), sodium pyruvate (Invitrogen;

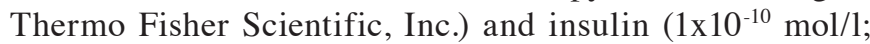
Invitrogen; Thermo Fisher Scientific, Inc.) at $37^{\circ} \mathrm{C}$ in humidified $5 \% \mathrm{CO}_{2}$.

Generation of stably overexpressing and resistant cell lines. MCF-7 cells were stably transfected with truncated CXCR4 ( $\triangle \mathrm{CTD}$ ), wild type CXCR4 (CXCR4), or empty vector as controls, as previously described (27). Fulvestrant resistant MCF-7N cells were generated by exposing the cells to 
gradually increasing concentrations of fulvestrant, until resistance was achieved, as described by Fan et al (38).

mRNA isolation. Cells were plated in 10\% DMEM at $70 \%$ confluency harvested after $24 \mathrm{~h}$ using a mix of phosphate-buffered saline and EDTA. Total RNA was isolated using the RNeasy kit, according to manufacturer's instructions (Qiagen, Inc.). Quantity and quality of RNA were determined by absorbance at 260 and $280 \mathrm{~nm}$ using the ND-1000 (NanoDrop).

Analysis of oligo-array data. Published oligo-array data by Ueda et al was analyzed using GeneGo Metacore (Thomson Reuters) (27). The Enrichment Analysis Workflow was performed using the gene list, fold-change, and P-value scores generated by edgeR. A threshold P-value of $<0.05$, and threshold fold-change $<0.5$ was set when performing the analysis in GeneGo.

The Published Kinase Inhibitor Sets (PKIS). The PKIS1 and PKIS2 are first generation kinase chemogenomic sets. They have now been supplanted by the KCGS (Kinase Chemogenomic Set) which is openly available in screening quantities from the SGC-UNC. Instructions for Requesting KCGS can be found at www.sgc-unc.org. Chemical structures and other pharmacologic activity for the PKIS compounds can be found at https://www.ebi.ac.uk/chembldb/extra/PKIS/compounds. html. The set is typically provided as $1 \mu \mathrm{l}$ of a $10 \mathrm{mM}$ solution in DMSO, dispensed in 384-well plates. A material transfer agreement was created to ensure that the screening results are made publicly available. Larger aliquots of requested compounds were delivered as solids, dissolved in DMSO to a $1 \mathrm{mM}$ stock solution, and stored at $-20^{\circ} \mathrm{C}$. The solutions were diluted in culture media and used at $1 \mu \mathrm{M}$ concentrations, as determined by dose-response studies.

Crystal violet staining. MDA-MB-157, MDA-MB-231, BT549, MCF-7-CXCR4- $\triangle$ CTD and MCF-7-FR cells were plated in a 96-well plate format at 2,000 cells per well. After $24 \mathrm{~h}$, cells were exposed to $5 \%$ charcoal stripped FBS media or phenol-free DMEM media (Invitrogen; Thermo Fisher Scientific, Inc.) supplemented with charcoal-stripped FBS, NEAA, MEM amino acids, Gluta-Max and penicillin (100 U/ml). After $48 \mathrm{~h}$ of exposing the cells to CS DMEM media, cells were treated with the vehicle or selected PKIS library compounds for $72 \mathrm{~h}$ and the plate was incubated in $37^{\circ} \mathrm{C}, 5 \% \mathrm{CO}_{2}$. The plate was then harvested by adding glutaraldehyde $(10 \mu \mathrm{l}$ of $25 \%$ stock solution) to each well for $20 \mathrm{~min}$. After rinsing and drying the plate, the cells were stained with $0.1 \%$ crystal violet in $90 \%$ methanol $(50 \mu \mathrm{l})$ for $20 \mathrm{~min}$. After another rinse, the cells were left overnight to dry, and the following day morphological alterations of the cells were visualized with an inverted microscope and images were recorded at x200 magnification.

\section{Results}

Candidate kinases identified that are responsible for promoting a mesenchymal phenotype in constitutive CXCR4 activation. Cell morphology and cytoskeletal rearrangement have important roles in suppressing metastasis, as epithelial-like cells are not able to invade and migrate into the vasculature to spread to distal tissue sites $(25,26)$. Mesenchymal morphology characteristics include bipolar cells often with protrusions that appear fibroblast-like, with minimal cell-cell contacts. Epithelial morphology cells have rounder shapes, increased circularity and form closer cell-cell contacts that facilitate colony formation and look more 'cobblestone' in appearance. Because some TNBC cell lines have inherently mesenchymal cell morphologies due to these cells' fibroblast-like characteristics, we chose to use three classic mesenchymal lines as positive controls in our phenotypic screen: MDA-MB-231, BT-549, MDA-MB-157. Only compounds were selected as 'hits' if they promoted an epithelial morphology in one or more TNBC cell lines and if they altered the morphology of MCF-7-CXCR4- $\triangle \mathrm{CTD}$ cells (Fig. 1A). Overall, we observed four different chemotypes that contained active and inactive compounds within the same chemotype, in which we could compare kinase activity. The four chemotypes were: Benzimidazole-N-thiophenes (Fig. 1B), oxindoles (Fig. S1), 4-hydrazinyl-pyrazolopyrimidines (Fig. S2), and furopyrimidines (Fig. S3).

Compounds in the PKIS library are non-selective kinase inhibitors, and thus they have target various kinases in addition to activity at the kinase for which they were originally designed. Many of the compounds in the PKIS library have kinase activity data described in a study by Elkins et al (2). Using these data sets we compared kinase activity of compound 'hits', referred to as active compounds, to activity of inactive compounds to find potential candidate kinases responsible for the observed phenotypic changes. In this first screen, active compounds altered cell morphologies and reversed the mesenchymal phenotype in MCF-7-CXCR4- $\triangle \mathrm{CTD}$ and TNBC cells, while inactive compounds did not. For these analyses, we compared active and inactive compounds that were within four chemotypes that had available published kinase activity data sets. Within the oxindole chemotype series, only one compound was active based on our initial screen, out of the 19 tested inhibitors. When kinase activity was compared in the active and inactive compounds, the only kinase which the active compound exhibited anti-kinase activity was DYRK2 (52\% anti-kinase activity). The inactive compounds in this series exhibited less anti-kinase activity against DYRK2: GW305178A (36\%), GW300660A (33\%), GR105659A (25\%), GW429374A (16\%), GW290597A (16\%), GW406108X (14\%), GW284408A (11\%), GW275616A (10\%), GW300657A (8\%), GW301789A (7\%), GW416469A (5\%), GW442130A (5\%), GW335962A (4\%), GW441756A (4\%), GW282536A (3\%), GW279320A (2\%), GW300653A (0\%), GW352430A (-1\%), GW278681A (-1\%). Within the benzimidazole $\mathrm{N}$-thiophenes chemotype series, 7 of the 13 compounds were active. The only kinase which the active compounds exhibited anti-kinase activity compared to inactive compounds was NEK9: GSK579289A (97\%), GSK237701A (92\%), GSK317315A (84\%), GW843682X (59\%), GW852849X (55\%), GSK237700A (38\%), GW853606X (29\%). Anti-NEK9 kinase activity of the inactive compounds includes: GW853609X(10\%), GSK1030061A(9\%), GSK1030058A(7\%), GSK1030059A (6\%), GSK1030062A (3\%), GSK319347A (2\%). Within the 4-hydrazinly-pyrazolopyrimidines chemotype series, 2 out of 8 compounds tested were active based on the initial screen. Kinases specific for the active compounds 

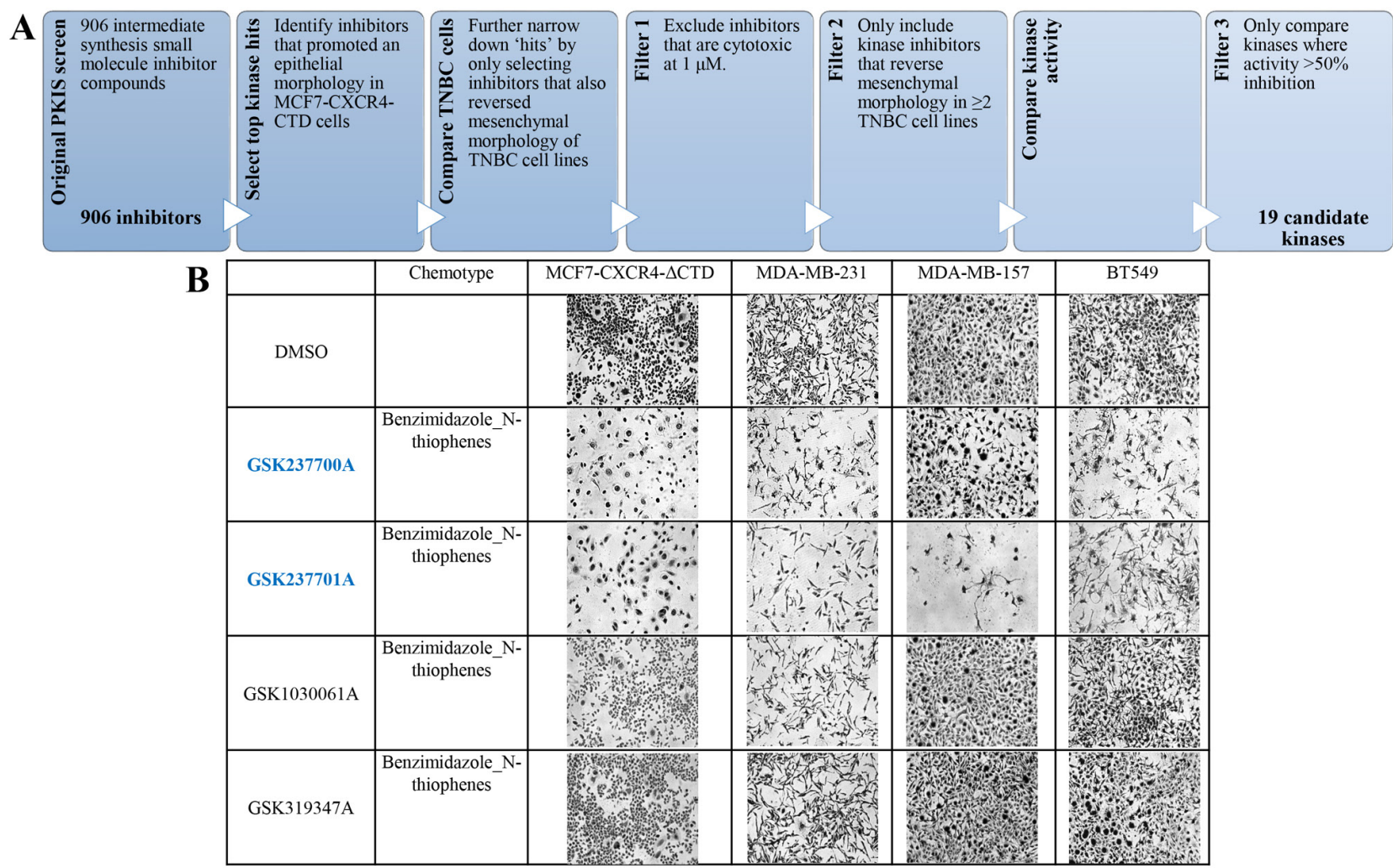

Figure 1. Identification of small molecule inhibitors from the PKIS library that reversed mesenchymal morphology and/or proliferation in both MCF-7-CXCR4- $\triangle$ CTD cells and TNBC cell lines. (A) Diagram showing workflow of medium-throughput phenotypic morphology screen using the PKIS library. (B) Select inhibitors that altered morphology in both the MCF-7-CXCR4- $\triangle$ CTD cells and at least one of the TNBC cell lines (MDA-MB-231, MDA-MB-157 and BT-549). An example of a chemotype of inhibitors in which some compounds affected morphology is represented, but some compounds had no effect on any of the cell lines analyzed. Compounds in blue were 'hits' in the screen. All cells were treated with $1 \mu \mathrm{M}$ inhibitor for $72 \mathrm{~h}$. Images were obtained at a magnification of x200 using brightfield microscopy. $\triangle \mathrm{CTD}$, truncated COOH-terminal domain; TNBC, triple-negative breast cancer; PKIS, Published Kinase Inhibitor Set; CXCR4, chemokine receptor 4.

\section{A Chemotype: 4-hydrazinly_pyrazolopyrimidines}

\begin{tabular}{rlllllllr} 
MAP4K4 & 78 & 75 & 8 & 35 & 38 & 17 & 44 & 21 \\
CDK2/cyclinA & 90 & 74 & 0 & 39 & 28 & 8 & 42 & 11 \\
CDK1/cyclinB & 77 & 74 & 15 & 33 & 16 & 1 & 23 & 8 \\
CDK5/p35 & 74 & 73 & 16 & 34 & 16 & 0 & 13 & 7 \\
SNF1LK2 (QIK) & 76 & 67 & -14 & 14 & 33 & 34 & 21 & 15 \\
SNF1LK1 (SIK) & 61 & 49 & -4 & 12 & 27 & 12 & 10 & 13 \\
MINK & 65 & 62 & 5 & 26 & 25 & 17 & 36 & 16 \\
PDGFRa-T674I & 78 & 82 & 1 & 22 & 17 & 18 & 38 & 17 \\
FYN & 84 & 59 & 5 & 32 & 41 & 26 & 53 & 16 \\
\hline
\end{tabular}

B Chemotype: Furopyrimidines_and_related

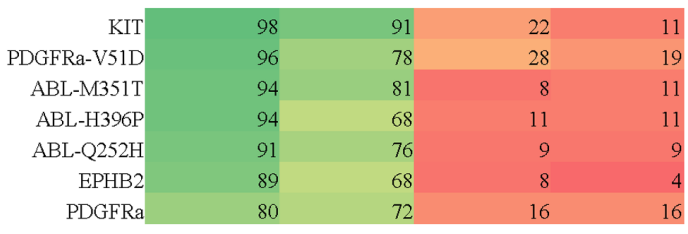

Figure 2. Candidate kinase targets activated by chemokine receptor 4 in the acquisition of a mesenchymal phenotype in triple-negative breast cancer identified by comparing the kinase activity of active and inactive compounds. Kinases selectively inhibited by 'active' compounds alone compared with 'inactive' compounds in the same chemotype. Heat map of (A) the 4-hydrazinyl pyrazolopyrimidine chemotype and (B) the furopyrimidine chemotype classes. Percent kinase activity is represented in the heat maps, with green indicating high activity and red low activity. Compounds in blue were identified as 'hits' in the screen. All cells were treated at $1 \mu \mathrm{M}$ inhibitor for $72 \mathrm{~h}$. 
A

\begin{tabular}{|c|c|c|c|}
\hline & Chemotype & MCF7-CXCR4- $\triangle$ CTD & MCF7-FR \\
\hline DMSO & -- & (n) & Th: \\
\hline GW814408X & \begin{tabular}{|c|}
$4-$ \\
hydrazinly_pyrazolopy \\
rimidines
\end{tabular} & (3) & \\
\hline GW643971X & \begin{tabular}{|c|}
$4-$ \\
hydrazinly_pyrazolopy \\
rimidines
\end{tabular} & 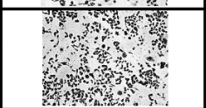 & tis. \\
\hline GW644007X & \begin{tabular}{|c|}
$4-$ \\
hydrazinly_pyrazolopy \\
rimidines
\end{tabular} & 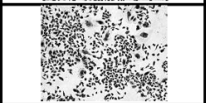 & tix \\
\hline GW578748X & \begin{tabular}{|c|}
$4-$ \\
hydrazinly_pyrazolopy \\
rimidines
\end{tabular} & 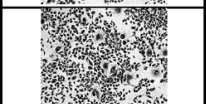 & 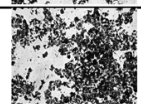 \\
\hline GW794607X & \begin{tabular}{|c|}
$4-$ \\
hydrazinly_pyrazolopy \\
rimidines
\end{tabular} & $\begin{array}{c}1 \\
4 \\
4\end{array}$ & 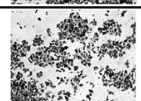 \\
\hline GW813360X & \begin{tabular}{|c|}
$4-$ \\
hydrazinly_pyrazolopy \\
rimidines
\end{tabular} & 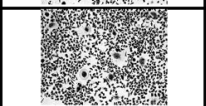 & 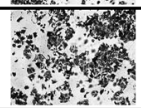 \\
\hline
\end{tabular}

B

\begin{tabular}{|c|c|c|c|}
\hline & Chemotype & MCF7-CXCR4- $\triangle \mathrm{CTD}$ & MCF7-FR \\
\hline GSK1173862A & $\begin{array}{c}2,4- \\
\text { dianilino_pyrrolop } \\
\text { yrimidines }\end{array}$ & 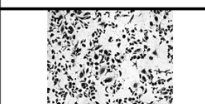 & 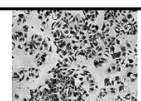 \\
\hline GSK994854A & $\begin{array}{c}2,4- \\
\text { dianilino pyrrolop } \\
\text { yrimidines }\end{array}$ & $\begin{array}{l}3 \\
40 \\
4\end{array}$ & $\begin{array}{l}3 \\
-3 \\
8\end{array}$ \\
\hline GSK2213727A & \begin{tabular}{|c|}
$2,4-$ \\
dianilino pyrrolop \\
yrimidines
\end{tabular} & & \\
\hline GSK2163632A & $\begin{array}{c}2,4- \\
\text { dianilino_pyrrolop } \\
\text { yrimidines }\end{array}$ & (3) & का \\
\hline GSK1392956A & $\begin{array}{c}2,4- \\
\text { dianilino_pyrrolop } \\
\text { yrimidines }\end{array}$ & 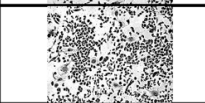 & \\
\hline GSK2219385A & $\begin{array}{c}2,4- \\
\text { dianilino_pyrrolop } \\
\text { yrimidines }\end{array}$ & ast & \\
\hline GSK1511931A & $\begin{array}{c}2,4- \\
\text { dianilino pyrrolop } \\
\text { yrimidines }\end{array}$ & 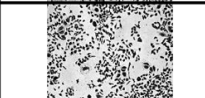 & 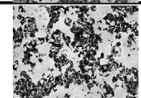 \\
\hline GSK1819799A & \begin{tabular}{|c|}
$2,4-$ \\
dianilino pyrrolop \\
yrimidines
\end{tabular} & 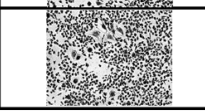 & 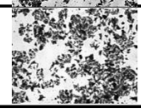 \\
\hline
\end{tabular}

Figure 3. Small molecule inhibitors from the Published Kinase Inhibitor Set library that affected cell morphology and/or proliferation in MCF-7-CXCR4- $\triangle \mathrm{CTD}$ cells compared with MCF-7-FR cells. Select inhibitors within (A) the 4-hydrazinly pyrazolopyrimidines or (B) the 2,4-dianilino pyrrolopyrimidines chemotype series that altered morphology in the MCF-7-CXCR4- $\triangle$ CTD cells and the MCF-7-FR cells. Inhibitors that were considered 'hits' altered cell morphology and/or cell proliferation. Compounds in blue were identified as 'hits' in the screen. $\triangle \mathrm{CTD}$, truncated COOH-terminal domain; CXCR4, chemokine receptor 4; FR, fulvestrant-resistant.

series, 2 out of 4 inhibitors tested in the initial screen were active. Kinases specific for active compounds compared to inactive compounds included: PDGFR $\alpha$-V561D, ABL-M351T, ABL-H396P, ABL-Q252H, EPHB2, PDGFR $\alpha$ (Fig. 2B). Together, these data demonstrate the utility of this small molecule inhibitor phenotypic screen approach and comparing kinase activity of active and inactive compounds to identify candidate kinases downstream of CXCR4 activation.

Use of phenotypically mesenchymal MCF-7 cells to identify candidate kinases that promote a mesenchymal and fulvestrant resistant phenotype in the setting of constitutive CXCR4 activation. MCF-7-FR cells have acquired resistance to fulvestrant and exhibit a mesenchymal cell phenotype (38). Here, we utilized these cells as another positive control in our screen to find candidate kinase targets that reversed the mesenchymal phenotype in addition to targets responsible for acquisition of an endocrine resistant phenotype (using cell viability as an endpoint) in CXCR4 activated MCF-7 cells. We discovered active inhibitors within the PKIS set that promoted an epithelial morphology and/or reduced cell viability in MCF-7-CXCR- $\triangle$ CTD cells and MCF-7-FR cells (Fig. 3A and B). Then we compared kinase activity of active and inactive compounds within the same chemotype series. Within the 2,4-dianilino pyrrolopyrimidines chemotype series 4 out of 8 tested inhibitors were active and ROS was the only kinase which active compounds targeted compared to inactive compounds (Fig. S4). Active compound kinase activity includes GSK2213727A (86\%), GSK2163632A (83\%), GSK1173862A (81\%), GSK994854A (61\%). Inactive compounds ROS kinase activity includes GSK1392956A (60\%), GSK1819799A (55\%), GSK2219385A (50\%), GSK1511931A (45\%). Within the 2-aryl-3-pridimidinyl pyrazolopyridazines chemotype series one out of 5 screened inhibitors were active (Fig. S5), within the 3-amino pyrazolopyridines series one out of 6 screened inhibitors were active (Fig. S6), within the 4-hydrazinly pyrazolopyrimidines group one out of 6 was an active inhibitor, within the maleimide chemotype series, one out of 10 screened inhibitors was active (Fig. S7) and in the furopyrimidines and related series two out of four tested inhibitors were active (Fig. S8).

In this phenotypic screen comparing the CXCR4 activated MCF-7 cells to fulvestrant resistant cells, kinases that were targeted by the active compounds and not in the inactive compounds included LYNA, CK1-g3, EPHB2, PDGFR $\alpha$-V561D, HCK, RET-Y791F, MINK, KIT-V560G (Fig. 4A), LTK, ABL variants, PIM1, PIM2, PIM3, CDK2, ALK, KDR, RSK3, BRSK1, BRSK2, MINK, PDGFR $\alpha$ (Fig. 4B), PDGFR $\alpha$-T, QIK, CDK1, CDK2, CDK5 (Fig. 4C) and other kinases (Fig. S9). Notably MAP4K4, HCK and ABL, PDGFR, PIM, and CDK family members were commonly targeted by active compounds amongst the various chemotype series (Figs. 4A-C and S9).

Then we compared results from the morphology screen (MCF7-CXCR4- $\triangle$ CTD compared to phenotypically mesenchymal TNBC cells) and viability (comparing MCF7-CXCR4- $\triangle$ CTD and MCF7-FR) screens. Eleven kinases were commonly targeted by active compounds in both screens compared to inactive compounds, suggesting these kinases were possibly responsible for both affecting the mesenchymal cell morphologies and sensitivity to endocrine-targeted therapies. These kinases included: MAP4K4, MINK, PDGFR $\alpha-V 561 D$, ABL-Q252H, PDGFR $\alpha$, SNF2LK(QIK), PDGFR $\alpha$-T351I, ABL-M351T, 
A Chemotype: 2-aryl 3-pridimidinyl pyrazolopyridazines

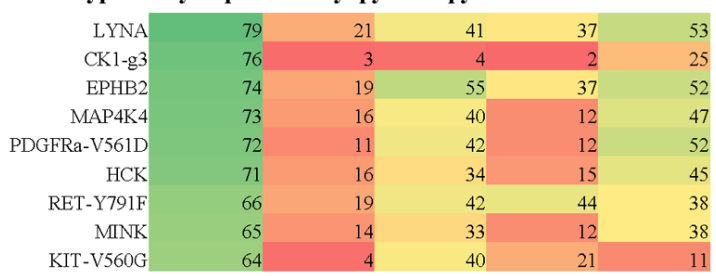

GW827099X GW827396X GW829055X GW828525X GW829906X

\section{Chemotype: 4-hydrazinly_pyrazolopyrimidines}

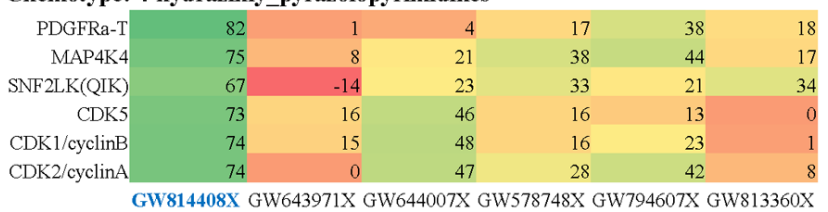

B Chemotype: 3-amino pyrazolopyridines

$\begin{array}{rrrrrrr}\text { LTK } & 92 & 1 & 46 & 1 & 1 & 1 \\ \text { ABLT315I } & 88 & 21 & 16 & 27 & 23 & 13 \\ \text { ABLQ252H } & 82 & 14 & 16 & 32 & 12 & 9 \\ \text { ABL-H396P } & 81 & 28 & 14 & 26 & 8 & 10 \\ \text { ABL-M351T } & 81 & 27 & 10 & 30 & 5 & 15 \\ \text { MAP4K4 } & 75 & 33 & 22 & 9 & 10 & 32 \\ \text { PII2 } & 75 & 0 & 16 & 0 & 1 & 0 \\ \text { PIM3 } & 74 & 1 & 45 & -3 & 6 & 0 \\ \text { ABL1 } & 73 & 19 & 11 & 24 & 8 & 14 \\ \text { CDK2/cyclinE } & 73 & 5 & 33 & 9 & 5 & 4 \\ \text { ALK } & 72 & 2 & 35 & 4 & -1 & 3 \\ \text { KDR } & 72 & 3 & 23 & 15 & 16 & 14 \\ \text { RSS3 } & 71 & 2 & 6 & 3 & 0 & 1 \\ \text { BRSK2 } & 70 & 11 & 27 & 11 & 19 & 5 \\ \text { MINK } & 68 & 22 & 15 & 8 & 6 & 18 \\ \text { ABL-Y253F } & 67 & 10 & 10 & 12 & 5 & 7 \\ \text { PDGFRa } & 67 & 12 & 21 & 13 & 5 & 11 \\ \text { BRSK1 } & 67 & 5 & 26 & 13 & 22 & 3 \\ \text { PIM1 } & 66 & -1 & 33 & 1 & 4 & 1 \\ \text { ABL-E255K } & 66 & 9 & 7 & 9 & 2 & 6 \\ \text { SB-725317 SB-711237 } & \text { SB-732881-H SB-739452 } & \text { SB-739245-AC SB-743899 }\end{array}$

Figure 4. Candidate kinase targets activated by chemokine receptor 4 that promote an endocrine resistant phenotype in breast cancer identified by comparing the kinase activity of active and inactive compounds. Kinase targets specific for active compounds (blue text) compared with inactive compounds in the same chemotype series. (A) 2-aryl 3-pyridimidinyl pyrazolopyridazines, (B) 3-amino pyrazolopyridines and (C) 4-hydrazinyl pyrazolopyrimidine chemotypes. Percent kinase activity is represented by the heat maps, with green indicating high activity and red low activity.

A

\begin{tabular}{|c|c|c|}
\hline Kinase & Chemotype & Screen \\
\hline \multirow{5}{*}{ MAP4K4 } & & Mesenchymal morphology \\
\hline & 4-hydrazinly_pyrazolopyrimidines & $\mathrm{FR}$ \\
\hline & 2-aryl_3-pyridimidinyl_pyrazolopyridazines & FR \\
\hline & 3-amino pyrazolopyridines & $\overline{F R}$ \\
\hline & Maleimide & FR \\
\hline \multirow{5}{*}{ MINK } & 4-hydrazinly pyrazolopyrimidines & Mesenchymal morphology \\
\hline & 2-aryl 3-pyridimidinyl pyrazolopyridazines & $\mathrm{FR}$ \\
\hline & 3-amino pyrazolopyridines & FR \\
\hline & Maleimide & $\mathrm{FR}$ \\
\hline & Furopyrimidines and related & Mesenchymal morphology \\
\hline \multirow[t]{2}{*}{ PDGFR $\alpha$-V561D } & 2-aryl 3-pyridimidinyl pyrazolopyridazines & $\mathrm{FR}$ \\
\hline & Maleimide & $\overline{F R}$ \\
\hline \multirow{3}{*}{ ABL-Q252H } & Furopyrimidines and related & Mesenchymal morphology \\
\hline & 3-amino pyrazolopyridines & FR \\
\hline & Maleimide & FR \\
\hline \multirow{3}{*}{ PDGFR $\alpha$} & Furopyrimidines and related & Mesenchymal morphology \\
\hline & 3-amino pyrazolopyridines & $\mathrm{FR}$ \\
\hline & Maleimide & $\overline{F R}$ \\
\hline \multirow[b]{2}{*}{ SNF2LK (QIK) } & 4-hydrazinly pyrazolopyrimidines & Mesenchymal morphology \\
\hline & Maleimide & $\frac{F R}{F R}$ \\
\hline \multirow{3}{*}{ PDGFRo-T351I } & 4-hydrazinly pyrazolopyrimidines & Mesenchymal morphology \\
\hline & & FR \\
\hline & Maleimide & FR \\
\hline \multirow{2}{*}{ ABL-M351T } & Furopyrimidines and related & Mesenchymal morphology \\
\hline & 3-amino pyrazolopyridines & $\mathrm{FR}$ \\
\hline \multirow[t]{2}{*}{ ABL-H396P } & Furopyrimidines and related & Mesenchymal morphology \\
\hline & 3-amino pyrazolopyridines & FR \\
\hline $\mathrm{CDK} 2 /$ cyclinA & 4-hydrazinly_pyrazolopyrimidines & Mesenchymal morphology \\
\hline \multirow{2}{*}{$\mathrm{CDK} 5 / \mathrm{p} 35$} & 4-hydrazinly pyrazolopyrimidines & Mesenchymal morphology \\
\hline & 4-hyarazinly pyrazolopyrimiaines & $\mathrm{FR}$ \\
\hline $\mathrm{CDK} 1 /$ cyclinB & 4-hydrazinly_pyrazolopyrimidines & Mesenchymal morphology \\
\hline \multirow{2}{*}{ SNF1LK (SIK) } & 4-hydrazinly pyrazolopyrimidines & $\frac{\text { FR }}{\text { Mesenchymal morphology }}$ \\
\hline & Maleimide & Mesencingmal morpnorogy \\
\hline \multirow{2}{*}{ KIT } & Furopyrimidines and related & Mesenchymal morphology \\
\hline & Maleimide & FR \\
\hline \multirow{2}{*}{ EPHB2 } & Furopyrimidines and related & yymal morphology \\
\hline & 2-aryl 3-pyridimidinyl pyrazolopyridazine & $\overline{F R}$ \\
\hline
\end{tabular}

B

\begin{tabular}{|c|c|c|}
\hline Kinase & Chemotype & Screen \\
\hline \multirow{2}{*}{$\begin{array}{c}\text { NEK9 } \\
\text { DYRK2 }\end{array}$} & Benzimidazole_N-thiophenes & Mesenchymal morphology \\
\hline & Oxindole & Mesenchymal morphology \\
\hline Kinase & \multicolumn{2}{|c|}{ Chemotype } \\
\hline \multirow{2}{*}{ ROS } & \multicolumn{2}{|c|}{ 2,4-dianilino pyrrolopyrimidines } \\
\hline & \multicolumn{2}{|c|}{ Maleimide } \\
\hline \multirow{2}{*}{ LTK } & \multicolumn{2}{|c|}{$\frac{\text { 3-amino pyrazolopyridines }}{\text { Maleimide }}$} \\
\hline & \multicolumn{2}{|c|}{ Maleimide } \\
\hline LCK & \multicolumn{2}{|c|}{ 2-aryl_3-pyridimidinyl_pyrazolopyridazines } \\
\hline \multirow[t]{2}{*}{ HCK } & \\
\hline & \multicolumn{2}{|c|}{ Maleimide } \\
\hline \multirow[t]{2}{*}{ RET-Y791F } & \multicolumn{2}{|c|}{$\frac{\text { 2-aryl 3-pyridimidinyl pyrazolopyridazines }}{\text { Maleimide }}$} \\
\hline & & imide \\
\hline \multirow{2}{*}{ RSK3 } & \multicolumn{2}{|c|}{ 3-amino pyrazolopyridines } \\
\hline & \multicolumn{2}{|c|}{ Maleimide } \\
\hline \multirow{2}{*}{ BRSK2 } & \multicolumn{2}{|c|}{ 3-amino pyrazolopyridines } \\
\hline & \multicolumn{2}{|c|}{ Maleimide } \\
\hline \multirow{2}{*}{ BRSK1 } & \multicolumn{2}{|c|}{ 3-amino pyrazolopyridines } \\
\hline & \multicolumn{2}{|c|}{ Maleimide } \\
\hline KIT-V560G & \multicolumn{2}{|c|}{ 2-aryl_3-pyridimidinyl_pyrazolopyridazines } \\
\hline ABLT315I & \multicolumn{2}{|c|}{ 3-amino_pyrazolopyridines } \\
\hline PYK2 & \multicolumn{2}{|c|}{ Maleimide } \\
\hline RET-V791F & \multicolumn{2}{|c|}{ Maleimide } \\
\hline FLT3-D & \multicolumn{2}{|c|}{ Maleimide } \\
\hline PDGFRa-D & $\mathrm{Ma}$ & simide \\
\hline PDGFRb & & imide \\
\hline TNK1 & & simide \\
\hline RSK1 & & simide \\
\hline RSK2 & & cimide \\
\hline RSK4 & & imide \\
\hline P70S6K1 & & eimide \\
\hline AURORAC & & imide \\
\hline CHEK2 & & imide \\
\hline
\end{tabular}

Figure 5. Candidate kinase targets identified in both the morphology and fulvestrant resistance screens. (A) Candidate kinase targets that were common hits in both the morphology (blue) and fulvestrant resistance (green) screens. (B) Kinase targets that were unique hits in the morphology screen. (C) Kinase targets that were unique hits in the fulvestrant resistance screen. FR, fulvestrant resistance.

ABL-H386P, CDK2/cyclin A, CDK5/p35, CDK1/cyclin B, SNF1LK(SIK), KIT and EPHB2 (Fig. 5A). Kinases that were unique hits in the morphology screen were NEK9 and DYRK2 (Fig. 5B). Kinase targets that were unique hits in the fulvestrant resistance screen that had over $70 \%$ kinase activity in the active compounds were ROS, LYK, LCK, HCK, RET-Y791F, RSK3, BRSK2, BRSK1, KIT-V560G, ABL-T351I, PYK2, RET-V791F, FLT3-D, PDGFR $\alpha$-D, PDGFRb, TNK1, RSK1, RSK2, RSK4, P70S6K1, AURORAC and CHEK2 (Fig. 5C). 
A

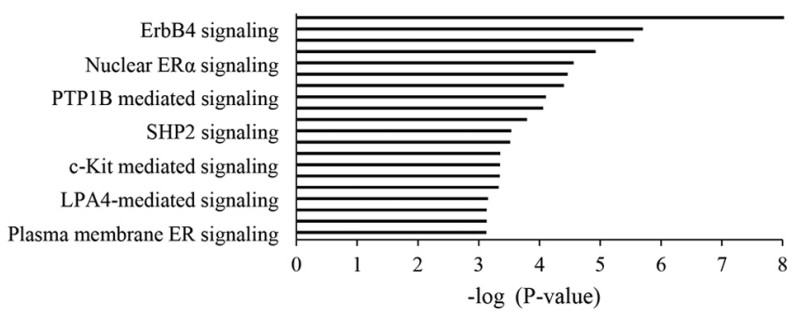

C

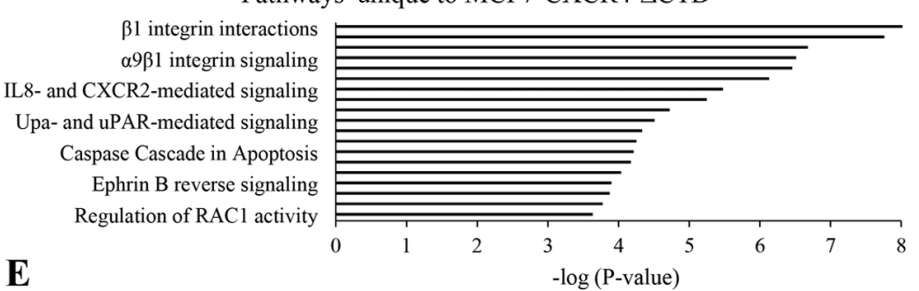

Genes upregulated in CXCR4-activated MCF-7 cells

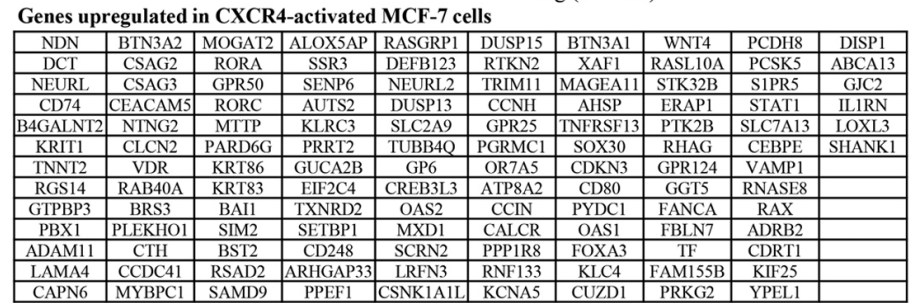

B

MCF7-CXCR4- $\triangle$ CTD pathways

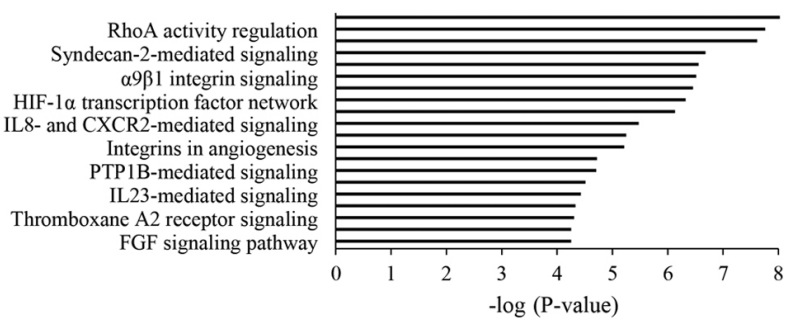

D

Pathways unique to MCF7-CXCR4

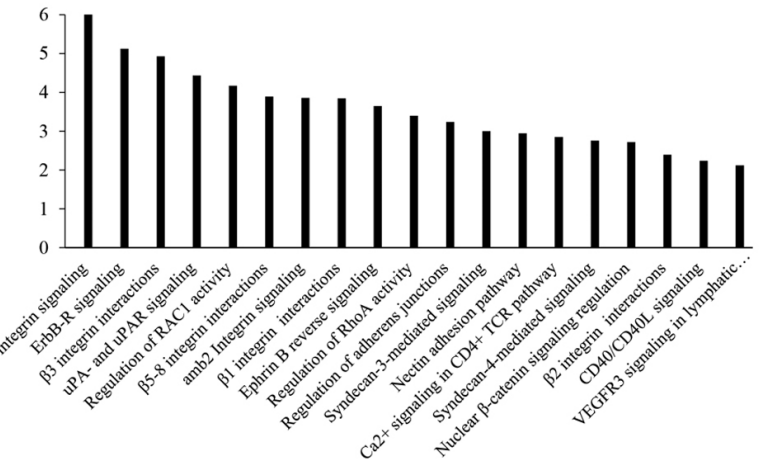

Figure 6. Downstream genes and pathways unique to the fulvestrant phenotype and CXCR4-activated phenotypes of MCF-7 cells. Analyses of oligoarray data were performed using the Gene Ontology enrichment analysis program. Pathway analysis data are shown as-log(P-value). (A) Genes upregulated in MCF-7-CXCR4 cells compared with empty vector controls (wild-type). (B) Genes upregulated in MCF-7-CXCR4- $\Delta$ CTD cells compared with empty vector controls. (C) Pathways unique to MCF-7-CXCR4- $\triangle$ CTD cells compared with MCF-7-CXCR4 cells. (D) Comprehensive list of pathways of selected genes with $>3$-fold difference in expression in MCF-7-CXCR4 cells compared with empty vector control cells. (E) List of specific genes upregulated in MCF-7-CXCR4 cells compared with empty vector control cells. $\triangle \mathrm{CTD}$, truncated $\mathrm{COOH}$-terminal domain; CXCR4, chemokine receptor 4.

Upregulated pathways regulated by CXCR4 identified through the screening approach were similar to those identified through oligoarrays. To compare potential mechanisms identified through our screen to other testing modalities, we performed a pathway analysis using oligo-array data provided by Ueda et al (27). For these analyses, we evaluated gene expression changes in MCF-7-CXCR4 and MCF-7-CXCR4- $\triangle$ CTD cells compared to the empty vector controls (MCF-7-VEC). We found that in CXCR4 overexpressing cells the most upregulated pathways included HDAC Class III, ERBB4, Endothelin, FOXA1, Nuclear ER $\alpha$, IL-2, and p75-mediated signaling pathways (Fig. 6A). In MCF-7-CXCR4- $\triangle$ CTD cells, there was an upregulation of $\beta 1$ integrin, RhoA, CXCR4-mediated, syndecan 2, LPA receptor and other integrin mediated signaling pathways (Fig. 6B). When pathways that were unique to MCF7-CXCR4- $\triangle$ CTD were examined, integrin-mediated signaling and RhoA signaling were within the top 10 most upregulated signaling pathways. Other signaling pathways included the uPA/uPAR, FAK, glypican 1, IL-8/CXCR2 and ephrin B-mediated signaling pathways (Fig. 6C). Pathways were then analyzed in genes that had over 3-fold difference in expression in MCF7-CXCR4 cells compared to vector control cells. In addition to integrin pathways, there was upregulation in ErbB receptor, ephrin B, adherent junctions and RhoA signaling pathways (Fig. 6D). Specific genes upregulated in CXCR4 activated cells were also shown in Fig. 6E. These data demonstrate potential downstream signaling mechanisms that CXCR4 employs to drive a mesenchymal phenotype.

\section{Discussion}

Estrogen independent hormone receptor positive breast cancers have acquired drug resistance, inhibiting response to endocrine-targeted therapies. Mesenchymal features of cancer cells that drive metastatic cancers are difficult to treat with currently available therapeutic regimens. In this proof-of-principle study, we introduce a new application of an available small molecule inhibitor chemogenomic library in a phenotypic screen approach to identify candidate kinases to pursue as potential therapeutic targets for the mesenchymal/metastatic phenotype and endocrine therapy resistance. Future investigations are required to interrogate these kinases and associated signaling pathways as potential downstream mechanisms of CXCR4.

Kinase profiling of the PKIS data sets using two endpoints (cell morphology and sensitivity to fulvestrant resistant cell lines) was used to identify known signaling pathways and novel candidate kinases responsible for the observed phenotypic and proliferative changes induced by constitutive CXCR4 activation. Kinases that reversed mesenchymal morphology compared to TNBC cell lines, were MINK, FYN, NEK9, and DYRK2. Kinases that were unique to the endocrine sensitivity screen were ROS, LCK, LYNA, p38 $\beta$, CK1-g3, LYNB, HCK, RET-Y791F, KIT-V560G, LTK, PIM2, PIM3, ABL1, CDK2/cyclinE, ALK, KDR, RSK3, BRSK2, BRSK1, PIM1, and ABL-E255K. The data suggests these kinases specifically affect the processes in which the 
CXCR4-activated cells either acquire a mesenchymal phenotype or acquire an endocrine therapy-resistant phenotype. We found another subset of kinases that were hits in both the morphology and fulvestrant resistant screens: MAP4K4, PDGFR $\alpha$-V561D, ABL-Q252H, PDGFR $\alpha$, SNF2LK(QIK), PDGFR $\alpha-T 351 \mathrm{I}$, ABL-M351T, ABL-H386P, CDK2/cyclinA, CDK1/cyclinB, CDK5/p35, SNF1LK(SIK), EPHB2, and KIT. Interestingly, three members of the CDK family were identified in our screen: CDK2/cyclinA, CDK1/cyclinB, CDK5/p35. While the role of CXCR4 in breast cancer metastasis is well established, Yi et al were the first to attempt to thoroughly interrogate possible downstream mechanisms of CXCR4 activity using phosphoproteomic-based methods (21). In their studies, CXCR4 substantially increased phosphorylation of CDK1, CDK3, and CDK7, which was novel because of the role for SDF-1/CXCR4 signaling in cell cycle regulation was not characterized (20). Here, our findings confirm a possible role for SDF-1/CXCR4 in CDK-mediated cell cycle processes.

Interestingly, variants in both the ABL and PDGFR $\alpha$ signaling pathways were within these kinase 'hits', indicating ABL and PDGFR $\alpha$ pathways have roles in CXCR4 downstream mechanisms. Crosstalk between ABL and CXCR4 signaling pathways exist through the Src kinase LYN $(39,40)$. Furthermore, other studies have shown ABL kinases are activated downstream of the CXCR4 receptor, facilitating ABL-mediated cell invasion and matrix degradation and, ultimately, metastasis (41). Our identification of ABL as a possible downstream kinase of CXCR4 activity in our screen in combination with these published studies, validates our approach.

Some of the candidate kinases identified in this screen had no previous associations to CXCR4 signaling. Examples of such kinases included MAP4K4, SNF2LK, SNF1LK, EPBH2, and KIT. Yi et al revealed a potential relationship between CXCR4 signaling and phosphorylation of MAP4K4 (21), but the mechanism behind this association has not yet been described. Here we further validate MAP4K4 as a candidate downstream kinase of CXCR4 activity to interrogate in future studies. SNF1LK and SNF2LK are serine/threonine kinases; SFK1LK, or SIK1, is downstream of LKB1, a well-described tumor suppressor protein. The association between SNF1LK and CXCR4 has not yet been reported. Ephrin B signaling was one of the pathways upregulated in MCF7-CXCR4-CTD cells, as identified in the oligoarray analyses. We further validate this finding in our small molecule inhibitor screen when EPHB2 was found to be a novel candidate downstream target of CXCR4 activity. EPHB2 is estrogen-independent, while other ephrin family members are estrogen dependent (42). Reverse signaling of ephrin B2 in endothelial cells is required for angiogenesis and is integral in metastasis $(42,43)$. Our data suggest that ephrin B signaling should be interrogated further as downstream regulators of CXCR4 function, and EPHB2 is the specific ephrin B family member on which to focus future mechanistic studies.

Employing another modality, such as an oligoarray, to assess potential mechanisms downstream of CXCR4 signaling validated the utility of our phenotypic screen approach. CXCR4 drives breast cancer metastasis by activating CXCR2 and MEK/PI3K pathways (28). Using oligoarrays, we found that CXCR4 overexpression increased expression of ERBB4 signaling as well as Rho/RAC signaling pathways. Similarly, in the screen within the top ten inhibitors that reversed the mesenchymal phenotype in TNBC cells in addition to altering the phenotypes of constitutively active CXCR4 cells, targets of these compounds included ERBB family members, GSK-3 $\beta$ and AKT.

The primary objective of this study was to demonstrate the utility of a comprehensive medium-throughput phenotypic screen using a readily available non-selective inhibitor set in a proof-of-concept study. A limitation of our study was that we only used one endocrine targeting therapy in the resistance screen, MCF-7 cells that were resistant to fulvestrant. We expect acquired resistance to other endocrine-targeting drugs to affect different kinase signaling pathways $(44,45)$. Another limitation was that there was only available off-target kinase comparison data for the PKIS1 library set. Because we screened PKIS1 and PKIS2 libraries, we hypothesized that comparing relative kinase activity in the PKIS2 set could lead to discovery of more targets, or validate the targets identified in the screen.

The interaction between SDF-1 and CXCR4 promotes a mesenchymal and migratory breast cancer cell phenotype, ultimately resulting in metastasis. SDF-1/CXCR4 signaling also facilities the acquisition of a resistant phenotype to endocrine targeting therapies. However, the mechanisms through which CXCR4 functions to promote this phenotype are not well characterized. In this study, using a phenotypic screen approach using the PKIS small molecule inhibitor set, we discovered candidate kinases and signaling pathways downstream of CXCR4 to be interrogated in future validation studies. This project provides valuable insight into novel mechanisms of CXCR4 activity and identifies potential pathways and targets to pursue using a comprehensive phenotypic screen approach. While our screening tool has promising preliminary findings, future projects are required to validate and interrogate the kinase pathway leads identified in this screen.

\section{Acknowledgements}

The authors would like to acknowledge the Structural Genomics Consortium (SGC), which provided the PKIS1 and PKIS2. The SGC is a registered charity (no. 1097737) that receives funds from AbbVie, Bayer Pharma AG, Boehringer Ingelheim, Canada Foundation for Innovation, Eshelman Institute for Innovation, Genome Canada, Innovative Medicines Initiative (EU/EFPIA), Janssen, Merck KGaA Darmstadt Germany, MSD, Novartis Pharma AG, Ontario Ministry of Economic Development and Innovation, Pfizer, Takeda and Wellcome (106169/ZZ14/Z). The authors would like to acknowledge Mr. Brandon Burow for his contributions in editing the manuscript.

\section{Funding}

The present study was funded by the National Institutes of Health (grant no. 1R01CA174785-01A1).

\section{Availability of data and materials}

All data generated or analyzed during this study are included in this published article. 


\section{Authors' contributions}

MDM wrote the first draft of the manuscript. MDM, SE, VTH and HEB performed data acquisition and analysis. LVR, ECM, WJZ, DHD, BMCB and MEB made substantial contributions to conception and design of the study, and interpretation of the data. MDM, WJZ, DHD and MEB were responsible for confirming the authenticity of the data. All authors provided revisions for the manuscript, read and approved the final manuscript.

\section{Ethics approval and consent to participate}

Not applicable.

\section{Patient consent for publication}

Not applicable.

\section{Competing interests}

The authors declare that they have no competing interests.

\section{References}

1. Eggert US: The why and how of phenotypic small-molecule screens. Nat Chem Biol 9: 206-209, 2013.

2. Elkins JM, Fedele V, Szklarz M, Abdul Azeez KR, Salah E, Mikolajczyk J, Romanov S, Sepetov N, Huang XP, Roth BL, et al: Comprehensive characterization of the published kinase inhibitor set. Nat Biotechnol 34: 95-103, 2016.

3. Drewry DH, Wells CI, Andrews DM, Angell R, Al-Ali H, Axtman AD, Capuzzi SJ, Elkins JM, Ettmayer $P$ Frederiksen M, et al: Progress towards a public chemogenomic set for protein kinases and a call for contributions. PLoS One 12: e0181585, 2017.

4. Matossian MD, Elliott S, Hoang VT, Burks HE, Phamduy TB, Chrisey DB,Zuercher WJ, Drewry DH, Wells C, Collins-Burrow B and Burrow ME: Novel application of the published kinase inhibitor set to identify therapeutic targets and pathways in triple negative breast cancer subtypes. PLoS One 12: e0177802, 2017.

5. Rubin JB: Chemokine signaling in cancer: One hump or two? Semin Cancer Biol 19: 116-122, 2009.

6. Balkwill FR: The chemokine system and cancer. J Pathol 226: 148-157, 2012.

7. Chatterjee S, Behnam Azad B and Nimmagadda S: The intricate role of CXCR4 in cancer. Adv Cancer Res 124: 31-82, 2014.

8. Bendall LJ, Baraz R, Juarez J, Shen W and Bradstock KF: Defective $\mathrm{p} 38$ mitogen-activated protein kinase signaling impairs chemotaxic but not proliferative responses to stromal-derived factor-1alpha in acute lymphoblastic leukemia. Cancer Res 65: 3290-3298, 2005.

9. Grande F, Giancotti G, Ioele G, Occhiuzzi MA and Garofalo A An update on small molecules targeting CXCR4 as starting points for the development of anti-cancer therapeutics. Eur J Med Chem 139: 519-530, 2017.

10. Walenkamp AME, Lapa C, Herrmann $\mathrm{K}$ and Wester HJ: CXCR4 Ligands: The next big hit? J Nuc Med 58 (Suppl 2):77S-82S, 2017.

11. Xu C, Zhao H, Chen H and Yao Q: CXCR4 in breast cancer: Oncogenic role and therapeutic targeting. Drug Des Devel Ther 9: 4953-4964, 2015.

12. Eckert F, Schilbach K, Klumpp L, Bardoscia L, Sezgin EC, Schwab M, Zips D and Huber SM: Potential role of CXCR4 targeting in the context of radiotherapy and immunotherapy of cancer. Front Immunol 9: 3018, 2018.

13. Mukherjee $\mathrm{D}$ and Zhao J: The role of chemokine receptor CXCR4 in breast cancer metastasis. Am J Cancer Res 3: 46-57, 2013.

14. Salvucci O, Bouchard A, Baccarelli A, Deschênes J, Sauter G, Simon R, Bianchi R and Basik M: The role of CXCR4 receptor expression in breast cancer: A large tissue microarray study. Breast Cancer Res Treat 97: 275-283, 2006.
15. Zhang Z, Ni C, Chen W, Wu P, Wang Z, Yin J, Huang J and Qiu F: Expression of CXCR4 and breast cancer prognosis: A systematic review and meta-analysis. BMC Cancer 14: 49, 2014.

16. Okuyama Kishima M, de Oliveira CE, Banin-Hirata BK, Losi-Guembarovski R, Brajão de Oliveira K, Amarante MK and Watanabe MA: Immunohistochemical expression of CXCR4 on breast cancer and its clinical significance. Anal Cell Pathol (Amst) 2015: 891020, 2015.

17. Chu QD, Panu L, Holm NT, Li BD, Johnson LW and Zhang S: High chemokine receptor CXCR4 level in triple negative breast cancer specimens predicts poor clinical outcome. J Surg Res 159: 689-695, 2010.

18. Hassan S, Ferrario C, Saragovi U, Quenneville L, Gaboury L, Baccarelli A, SalvucciO and Basik M: The influence of tumor-host interactions in the stromal cell-derived factor-1/CXCR4 ligand/receptor axis in determining metastatic risk in breast cancer. Am J Pathol 175: 66-73, 2009.

19. Krohn A, Song YH, Muehlberg F, Dross L, Bechmann C and Alt E: CXCR4 receptor positive spheroid forming cells are responsible for tumor invasion in vitro. Cancer Lett 280: 65-71, 2009.

20. Graham NA and Graeber TG: Complexity of metastasis-associated SDF-1 ligand signaling in breast cancer stem cells. Proc Natl Acad Sci USA 111: 7503-7504, 2014.

21. Yi T, Zhai B, Yu Y, Kiyotsugu Y, Raschle T, Etzkorn M, Seo HC, Nagiec M, Luna RE, Reinherz EL, et al: Quantitative phosphoproteomic analysis reveals system-wide signaling pathways downstream of SDF-1/CXCR4 in breast cancer stem cells. Proc Natl Acad Sci USA 111: E2182-E2190, 2014.

22. Mego M, Cholujova D, Minarik G, Sedlackova T, Gronesova P, Karaba M, Benca J, Cingelova S, Cierna Z, Manasova D, et al: CXCR4-SDF-1 interaction potentially mediates trafficking of circulating tumor cells in primary breast cancer. BMC Cancer 16: 127,2016

23. Brabletz T, Kalluri R, Nieto MA and Weinberg RA: EMT in cancer. Nat Rev Cancer 18: 128-134, 2018.

24. Felipe Lima J, Nofech-Mozes S, Bayani J and Bartlett JM: EMT in breast carcinoma-A review. J Clin Med 5: 65, 2016.

25. Leggett SE, Sim JY, Rubins JE, Neronha ZJ, Williams EK and Wong IY: Morphological single cell profiling of the epithelial-mesenchymal transition. Integr Biol (Camb) 8: 1133-1144, 2016.

26. Nelson CM, Khauv D, Bissell MJ and Radisky DC: Change in cell shape is required for matrix metalloproteinase-induced epithelial-mesenchymal transition of mammary epithelial cells. J Cell Biochem 105: 25-33, 2008.

27. Ueda Y, Neel NF, Schutyser E, Raman D and Richmond A: Deletion of the COOH-terminal domain of CXC chemokine receptor 4 leads to the down-regulation of cell-to-cell contact, enhanced motility and proliferation in breast carcinoma cells. Cancer Res 66: 5665-5675, 2006.

28. Sobolik T, Su YJ, Wells S, Ayers GD, Cook RS and Richmond A: CXCR4 drives the metastatic phenotype in breast cancer through induction of CXCR2 and activation of MEK and PI3K pathways. Mol Biol Cell 25: 566-582, 2014

29. Sun X, Cheng G, Hao M, Zheng J, Zhou X, Zhang J, Taichman RS, Pienta KJ and Wang J: CXCL12/CXCR4/CXCR7 chemokine axis and cancer progression. Cancer Metastasis Rev 29: 709-722, 2010.

30. Rhodes LR, Short SP, Neel NF, Salvo VA, Zhu Y, Elliott S, Wei Y, Yu D, Sun M, Muir SE, et al: Cytokine receptor CXCR4 mediates estrogen-independent tumorigenesis, metastasis, and resistance to endocrine therapy in human breast cancer. Cancer Res 71: 603-613, 2010

31. Liang S, Peng X, Li X, Yang P, Xie L, Li Y, Du C and Zhang F: Silencing of CXCR4 sensitizes triple-negative breast cancer cells to cisplatin. Oncotarget 6: 1020-1030, 2015.

32. Wang Y, Yan L, Zhang L, Xu H, Chen T, Li Y, Wang H, Chen S, Wang W, Chen C and Yang Q: NT21MP negatively regulates paclitaxel-resistant cells by targeting miR-155-3p and miR-155-5p via the CXCR4 pathway in breast cancer. Int J Oncol 53: 1043-1054, 2018.

33. Liu S, Xie SM, Yang-Kolodji G and Tripathy D: Targeting the tumor microenviroment by CXCR4 inhibition to abrogate trastuzumab resistance in HER2-positive breast cancer. Cancer Res 79 (Suppl 4): P5-03-04, 2019.

34. Rhodes LV, Antoon JW, Muir SE, Elliott S, Beckman BS and Burrow ME: Effects of human mesenchymal stem cells on ER-positive human breast carcinoma cells mediated through ER-SDF-1/CXCR4 crosstalk. Mol Cancer 9: 295, 2010. 
35. Rhodes LV, Bratton MR, Zhu Y, Tilghman SL, Muir SE, Salvo VA, Tate CR, Elliott S, Nephew KP, Collins-Burow BM and Burow ME: Effects of SDF-1-CXCR4 signaling on microRNA expression and tumorigenesis in estrogen receptor-alpha (ER- $\alpha$ )-positive breast cancer cells. Exp Cell Res 317: 2573-2581, 2011.

36. Sauvé K, Lepage J, Sanchez M, Heveker N and Tremblay A: Positive feedback activation of estrogen receptors by the CXCL12-CXCR4 pathway. Cancer Res 69: 5793-5800, 2009.

37. Dubrovska A, Hartung A, Bouchez LC, Walker JR, Reddy VA, Cho CY and Schultz PG: CXCR4 activation maintains a stem cell population in tamoxifen-resistant breast cancer cells through AhR signalling. British J Cancer 107: 43-52, 2012

38. Fan M, Yan PS, Hartman-Frey C, Chen L, Paik H, Oyer SL, Salisbury JD, Cheng AS, Li L, Abbosh PH, et al: Diverse gene expression and DNA methylation profiles correlate with differential adaptation of breast cancer cells to the antiestrogens tamoxifen and fulvestrant. Cancer Res 66: 11954-11966, 2006.

39. Ptasznik A, Urbanowska E, Chinta S, Costa MA, Katz BA, Stanislaus MA, Demir G, Linnekin D, Pan ZK and Gewirtz AM: Crosstalk between BCR/ABL oncoprotein and CXCR4 signaling through a Src family kinase in human leukemia cells. J Exp Med 196: 667-678, 2002.

40. Dillmann F, Veldwijk MR, Laufs S, Sperandio M, Calandra G, Wenz F, Zeller J and Fruehauf S: Plerixafor inhibits chemotaxis toward SDF-1 and CXCR4-mediated stroma contact in a dose-dependent manner resulting in increased susceptibility of BCR-ABL+ cell to Imatinib and Nilotinib. Leuk Lymphoma 50: 1676-1686, 2009.
41. Smith-Pearson PS, Greuber EK, Yogalingam G and Pendergast AM: Abl kinases are required for invadopodia formation and chemokine-induced invasion. J Biol Chem 285: 40201-40211, 2010.

42. Kaenel P, Mosimann M and Andres AC: The multifaceted roles of Eph/ephrin signaling in breast cancer. Cell Adh Migr 6: 138-147, 2012.

43. Haldimann M, Custer D, Munarini N, Stirnimann C, Zürcher G, Rohrbach V,Djonov V,Ziemiecki A and Andres AC: Deregulated ephrin-B2 expression in the mammary gland interferes with the development of both the glandular epithelium and vasculature and promotes metastasis formation. Int $\mathrm{J}$ Oncol 35: 525-536, 2009.

44. Lamb CA, Helguero LA, Fabris V, Lucas C, Molinolo AA and Lanari C: Differential effects of raloxifene, tamoxifen and fulvestrant on a murine mammary carcinoma. Breast Cancer Res Treat 79: 25-35, 2003.

45. Giuliano M, Schiff R, Osborne CK and Trivedi MV: Biological mechanisms and clinical implications of endocrine resistance in breast cancer. Breast 20 (Suppl 3): S42-S49, 2011. International (CC BY-NC-ND 4.0) License. 